\title{
Anatomy of the coronary artery and cardiac vein in the quail ventricle: patterns are distinct from those in mouse and human hearts
}

Masahiro Kato, Mayu Narematsu, Yuji Nakajima

\begin{tabular}{|c|l|}
\hline Citation & Anatomical Science International, 93(4): $533-539$ \\
\hline Issue Date & 2018-09 \\
\hline Type & Journal Article \\
\hline Textversion & author \\
\hline Rights & $\begin{array}{l}\text { This is a post-peer-review, pre-copyedit version of an article published in } \\
\text { Anatomical Science International. The final authenticated version is available } \\
\text { online at: } \text { https://doi.org/10.1007/s12565-018-0446-x }\end{array}$ \\
\hline DOI & $10.1007 / \mathrm{s} 12565-018-0446-\mathrm{x}$ \\
\hline
\end{tabular}

Self-Archiving by Author(s)

Placed on: Osaka City University

Kato, M., Narematsu, M. \& Nakajima, Y. (2018). Anatomy of the coronary artery and cardiac vein in the quail ventricle: patterns are distinct from those in mouse and human hearts. Anatomical Science International. 93: 533. doi:10.1007/s12565-018-0446-x 
1 Anatomy of the coronary artery and cardiac vein in the quail ventricle: patterns are distinct from

2 those in mouse and human hearts

3

4 Short title: Anatomy of quail coronary vessels

5

6 Masahiro Kato ${ }^{1,2}$, Mayu Narematsu ${ }^{2}$, Yuji Nakajima ${ }^{2}$

$7 \quad{ }^{1}$ Fifth-year medical student, Osaka City University, Osaka 545-8585 Japan

$8{ }^{2}$ Department of Anatomy and Cell Biology, Graduate School of Medicine, Osaka City University, Osaka

9 545-8585 Japan

10

11 Correspondence:

12 Yuji Nakajima MD, PhD

13 Department of Anatomy and Cell Biology, Graduate School of Medicine, Osaka City University

14 1-4-3 Asahimachi, Abenoku, Osaka 545-8585 Japan

$15 \quad$ yuji@med.osaka-cu.ac.jp

16 Tel: +81-6-6645-3705

17 Fax: +81-6-6646-3603

18 
Coronary vessel development has been investigated in avian and mouse embryonic hearts. Quail embryos are a useful tool to examine vascular development, particularly because the QH1 antibody and transgenic quail line, Tg (tie1:H2B-eYFP), are useful to trace endothelial cells. However, there are only a few descriptions of the quail coronary vessels. Using ink injection coronary angiography, we examined the course of coronary vessels in the fetal quail heart. The major coronary arteries were the right and left septal arteries, which respectively branched off from the right and left coronary stems. The right septal artery ran posteriorly (dorsally) and penetrated the ventricular free wall to distribute to the posterior surface of the ventricles. The left septal artery ran anteriorly (ventrally) and penetrated the ventricular free wall to distribute to the anterior surface of the ventricles. The right and left circumflex arteries were directed posteriorly along the atrioventricular sulci. The cardiac veins consisted of three major tributaries: the middle, great, and anterior cardiac veins. The middle cardiac vein ascended along the posterior interventricular sulcus and emptied into the right atrium. The great cardiac vein ran along the anterior interventricular sulcus, entered the space between the left atrium and conus arteriosus and emptied into the right atrium behind the aortic bulb. The anterior cardiac vein drained the anterior surface of the right ventricle and connected to the anterior base of the right atrium. The course of coronary vessels in the quail heart was basically the same as that observed in chick but was different from those of mouse and human. 
38 Key words: anatomy, avian species, cardiac vein, coronary artery, quail heart

40 Quail major coronary vessels consist of right and left septal arteries; and middle, great and anterior cardiac veins. Quail coronary patterns are distinct from those of mouse and human hearts.

\section{Introduction}

The coronary circulatory system is essential to maintain nutrients and oxygen provision for the cardiac muscle to function throughout life. The coronary circulation starts immediately after metamorphosis, way oxygen is supplied to the cardiac muscle changes dramatically, i.e. diffusion from the endocardium is replaced by the adult coronary circulatory system consisting of coronary arteries and veins. Impaired development of the coronary vessels leads to life-threatening defects, including aberrant origins of the coronary artery and coronary arteriovenous fistula (Pe'rez-Pomares et al. 2016). Coronary arteriosclerosis causes ischemic heart disease in adult life. Revascularization of the ischemic or infarcted lesions is a potential therapeutic strategy to restore cardiac contractility. Therefore, it is important to understand the mechanisms regulating coronary vessel formation in order to develop the revascularization therapies 
surface mesothelium and a sinus venosus-derived inner core mesenchyme, generates the coronary

demonstrated that the endothelial cells lining the sinus venosus and ventricles are the major sources of

arteries originate, respectively, from the right and left aortic sinuses. However, the patterns and courses of interventricular and coronary sulci subjacent to the epicardium, whereas mouse coronary arteries course the coronary stem, but there exist some differences in coronary patterning between birds (Myczkowski, vascular development, because the quail endothelium-specific antibody QH1 and transgenic quail model, Tg (tie1:H2B-eYFP), are available to trace endothelial cells (Pardanaud et al. 1987; Sato and Lansford 2013). However, there have been few published studies presenting the actual macroscopic anatomy of the quail coronary vessels (Fitzgerald 1969). In the present report, using ink injection coronary angiography we observed the origin and course of coronary arteries and veins in fetal quail heart. The major coronary arteries were the right and left septal arteries, which respectively branched off from the right and left 
coronary stems. Cardiac veins were classified into three major veins including the middle, great, and

anterior cardiac veins according to their opening into the right atrium.

Materials and Methods

Fertilized quail eggs (Quail COSMOS, Toyohashi, Japan) were incubated at $37.5^{\circ} \mathrm{C}$ and $60 \%$ humidity.

Embryos were staged by days of incubation (embryonic day, E) and using the Hamburger and Hamilton

system (1951) adapted into quail (Ainsworth et al. 2010), and E6 to 14 (stage 29 to 43) hearts were

examined. The number of embryos examined is tabulated in Table 1. Eggs were placed on ice for 30

minutes to stop the heart-beat, the embryo was extirpated and put in ice-cooled phosphate buffered saline

(PBS). The thoracic cavity was opened, then a pulled glass needle was inserted into the ascending aorta,

and $4 \%$ paraformaldehyde (PFA)/PBS was gently injected. To observe the coronary artery, $20 \%$ carbon ink (PLATINUM, Tokyo, Japan) in PBS was injected into the ascending aorta. The resulting hearts were

extirpated and re-fixed in $4 \% \mathrm{PFA} / \mathrm{PBS}$ at $4^{\circ} \mathrm{C}$ for more than 12 hours. The fixed hearts were immersed in a graded series of glycerin $/ 0.5 \%$ potassium hydroxide ( $1: 3,15$ minutes; $1: 1,30$ minutes; $3: 1,2$ hours) for transparency. To observe the cardiac vein, $20 \%$ carbon ink $/ 2 \%$ gelatin in PBS was injected into the ascending aorta, after the cardiac veins connecting to right atrium were visualized, the hearts were removed and re-fixed in ice-cooled 4\% PFA/PBS for more than 48 hours. Gelatin was used to keep the 


\section{Results}

\section{Coronary arteries}

The right coronary stem, which originated from the right aortic sinus, gave off surface and deep branches.

101 The surface arteries included the conus branch (cb in Fig. 1A), subepicardial branches of the right ventricular free wall (rvb in Fig. 1A), and the right circumflex branch (cx in Fig. 1A). The right circumflex branch ran along the right coronary sulcus. It gave off right atrial branches along the pectinate muscles as well as terminal branches on the right lateral surface of the right ventricle. The deep artery, the pierced the supraventricular crista to enter into the interventricular septum (right side of the yellow dotted interventricular septum and apex. The peripheries of the right septal artery penetrated the ventricular free wall transmurally at the posterior interventricular sulcus and distributed on the posterior surface of both ventricles and the apex (small arrows in Fig. 1C). 
114 thick artery, the left septal artery, pierced the infundibulum to enter the interventricular septum (sa in Fig.

115 1E) and gave off branches toward the anterior (ventral) aspect of the interventricular septum and apex

116 (left side of the yellow dotted line in Fig. 1E and gray area in Fig. 1E'). The peripheries of the left septal

117 artery penetrated the ventricular free wall and distributed onto the surface of the left ventricular free wall

118 and apex (small arrows in Fig. 1D and E).

119

120 Cardiac veins

121 Cardiac veins were classified into three major veins according to their openings into the right atrium, i.e.

middle cardiac vein, great cardiac vein, and anterior cardiac vein. The middle cardiac vein was the largest

vein and was observed in all samples examined (20/20) (mcv in Fig. 2A). The middle cardiac vein, which

drained from the posterior surface of both ventricles including the apex, ran along the posterior

125 interventricular sulcus and emptied into the base of the left precaval vein (left superior vena cava) before

126 E9 (stage 36-37) and emptied into the sinus venosus of the right atrium after E10 (stage 38-39). The left

127 circumflex vein (cx in Fig. 2A), which originated from the left lateral wall of the left ventricle and passed

128 into the left coronary sulcus, was observed in 14 hearts (14/20) and terminated at the left precaval vein

129 (5/14), right atrium (5/14) or middle cardiac vein (4/14). Small cardiac veins were observed in $5(5 / 20)$

130 hearts and joined into the middle cardiac vein (scv in Fig. 2A).

The great cardiac vein consisted of two major segments, the anterior interventricular segment and a 
133 the anterior interventricular sulcus (aivs in Fig. 2B) and entered the space between the pulmonary trunk

134 and left auricle to continue the basal segment (bs in Fig. 2B). The basal segment ran along the anterior

135 basal rim of the left atrium (accompanying the left coronary artery stem) and finally emptied into the right

136 atrium behind the base of the ascending aorta (open arrowhead in Fig. 2B). In 2 of 18 hearts, no apparent

137 interventricular segment was observed. The left conus vein was observed in all samples with a visible

138 great cardiac vein (18/18). A direct anastomosis between the great cardiac vein and left circumflex vein

139 was detected in 6 samples (6/18).

140 The anterior cardiac vein, which drained from the anterior wall of the right ventricle via several

141 tributaries and the right conus vein, emptied into the base of the right auricle (acv and open arrowhead in

142 Fig. 2C). The opening of the anterior cardiac vein had a single orifice (13/16; 3 openings were found in

143 one sample and undetectable in 2 samples).

144

145 Discussion

\section{Coronary artery}

147 In quail hearts, the main coronary arteries were the right and left septal arteries, which respectively

148 branched off from the right and left coronary stems and penetrated the supraventricular crista and

149 infundibulum to enter the interventricular septum. These deep arteries distributed into the interventricular

150 septum, subsequently penetrated the ventricular free wall to exit into the subepicardial space, and

151 distributed into the ventricular surface and apex (Fig. 3A). The anatomy of quail coronary arteries 
right coronary sulcus, giving off an acute marginal branch and turns obliquely toward the apex as a dorsal

Swiss albino mouse (Yoldas et al. 2010). The septal artery predominantly branches off from the right 
171 The quail cardiac vein system consisted of three cardiac veins according to their openings into the right

172 atrium: the middle cardiac vein, great cardiac vein, and anterior cardiac vein (Fig. 3). The patterns of

173 quail cardiac veins were similar to those observed in chick hearts (Lindsay 1967). The most characteristic

174 feature of the quail cardiac veins was the course of the great cardiac vein. In this vein, the anterior

175 interventricular segment ascended the lateral aspect of the interventricular sulcus followed by the basal

176 segment. The basal segment ran between the left atrium and conus arteriosus and opened solely into the

177 right atrium behind the aortic bulbs. This type of great cardiac vein is commonly observed in chicks ( $78 \%$

178 [60/78] Lindsay 1967), monotremes (Dowd 1969), and phalangers (marsupials) (Dowd 1974), but rarely

179 in pigs (Alejandro Gómez et al. 2015) and humans (0.3\% [1/337], Kawashima et al. 2003). The middle

180 cardiac vein is the largest cardiac vein in birds, and it drains from the apex and posterior ventricular

181 surface, ascends along the interventricular sulcus, and opens into the right atrium. The middle cardiac

182 vein is commonly observed in birds as well as in mammals, suggesting that the middle cardiac vein is

183 conserved across species. The anterior cardiac vein drained the anterior free wall of the right ventricle via

184 the right conal vein and several tributaries of the anterior ventricular wall. These tributaries united into a

185 single canal to open the anterior basal rim of the right auricle. The course and opening of the quail

186 anterior cardiac vein resembled with those in chicks and humans, in which one (most common) to three

187 anterior cardiac veins receive tributaries from the right ventricular free wall, empty the right atrium via

188 the luminal vein (Lindsay 1967; von Ludinghausen 1987; Loukas et al. 2009b).

189 The course of cardiac veins in the mouse heart resembles that in rats, but different from that in 
191 veins emptying to the coronary sinus have been identified. These include the left cardiac vein (the largest

192 cardiac vein and comparable to the human left marginal vein), caudal vein (middle cardiac vein), and

193 right cardiac vein (right marginal vein). There is no great cardiac vein running in the anterior

194 interventricular sulcus. The remarkable features of the mouse/rat cardiac vein are the conal veins, in

which the right conal vein courses on the ventral aspect of the left ventricular outflow tract and opens into

the right atrium, and the left conal vein runs behind the pulmonary trunk and connects with the right conal

vein, right atrium, or right cranial caval vein. Therefore, the conal veins surrounding the great arteries are

similar to the peritruncal endothelial plexus (peritruncal ring) observed in embryonic hearts (Ando et al.

2004). It may be suggested that the left conal vein passing behind the great arteries (pulmonary trunk and

\section{Coronary arteriovenous fistula}


210 (stage 38-39) onward. Therefore, the capillary beds interposing the arteries and veins may rapidly develop

211 after the coronary circulation starts. Coronary arteriovenous fistula (a type of congenital heart defect), in

212 which the right coronary artery connect the right atrium without a capillary bed (Pe'rez-Pomares et al.

213 2016), may occur due to the persistence of the direct connection of the coronary artery and cardiac vein.

214 We described the topographical anatomy of the quail coronary arteries and cardiac veins. The major

215 coronary arteries of the quail heart are the right and left septal arteries, which respectively originate from

216 the right ant and left coronary stems. The cardiac veins are classified into the middle, great, and anterior

217 cardiac veins according to their openings into the right atrium. The course of the quail coronary vessels

218 was basically the same as that in chick heart but was different from mouse and human hearts.

\section{Acknowledgments}

221 Authors thank S Uoya for preparing the manuscript. This work was supported by JSPS Grant-in-Aid

222 Scientific Research (c) 16K08450.

223

\section{Conflict of Interest}

The authors declare that they have no conflict of interest.

226 
Alejandro Gomez F, Ballesteros LE, Stella Cortes L (2015) Morphological description of great cardiac vein in pigs compared to human hearts. Braz J Cardiovasc Surg 30:63-69

Ando K, Nakajima Y, Yamagishi T, Yamamoto S, Nakamura H (2004) Development of proximal coronary arteries in quail embryonic heart: multiple capillaries penetrating the aortic sinus fuse to form main coronary trunk. Circ Res 94: 346-352

Bezuidenhout AJ (1984) The coronary circulation of the heart of the ostrich (Struthio camelus). J Anat

Ciszek B, Skubiszewska D, Ratajska A (2007) The anatomy of the cardiac veins in mice. J Anat

Dowd DA (1969) The coronary vessels and conducting system in the heart of monotremes. Acta Anat

Dowd DA (1974) The coronary vessels in the heart of a marsupial, Trichosurus vulpecula. Am J Anat 140: $47-56$ coronary arteries of the C57BL/6 mouse strains: implications for comparison with mutant models. J 
Hamburger V, Hamilton HL (1951) A series of normal stages in the development of the chick embryos. J

Kamimura T, Yamagishi T, Nakajima Y (2018) Avian coronary endothelium is a mosaic of sinus venosusand ventricle-derived endothelial cells in a region-specific manner. Dev Growth Differentiation $60: 97-111$

Kawashima T, Sato K, Sato F, Sasaki H (2003) An anatomical study of the human cardiac veins with special reference to the drainage of the great cardiac vein. Ann Anat 185: 535-542 Alonso-Briales JH, Fernandez B (2016) Unusual anatomical origins of the coronary arteries in 
Nakajima Y, Imanaka-Yoshida K (2013) New insights into the developmental mechanisms of coronary

Pardanaud L, Altmann C, Kitos P, Dieterlen-Lievre F, Buck CA (1987) Vasculogenesis in the early quail

Perez-Pomares JM, de la Pompa JL, Franco D, Henderson D, Ho SY, Houyel L, Kelly RG, Sedmera D, anomalies: a bridge from embryology to anatomy and pathophysiology--a position statement of the

Reese DE, Mikawa T, Bader DM (2002) Development of the coronary vessel system. Circ Res

Sato Y, Lansford R (2013) Transgenesis and imaging in birds, and available transgenic reporter lines. Dev 
284 Tomanek RJ, Hansen HK, Dedkov EI (2006) Vascular patterning of the quail coronary system during

285 development. Anat Rec A Discov Mol Cell Evol Biol 288: 989-999

286 M von Lüdinghausen M (1987) Clinical anatomy of cardiac veins, Vv. cardiacae. Surg Radiol Anat

$287 \quad 9: 159-168$

288 Vrancken Peeters MP, Gittenberger-de Groot AC, Mentink MM, Hungerford JE, Little CD, Poelmann RE

289 (1997) The development of the coronary vessels and their differentiation into arteries and veins in the

290 embryonic quail heart. Dev Dyn 208:338-348

291 Yoldas A, Ozmen E, Ozdemir V (2010) Macroscopic description of the coronary arteries in Swiss albino

292 mice (Mus musculus). J S Afr Vet Assoc 81: 247-252

293 
Right and left coronary arteries. A) Surface branches of the right coronary artery include the conus branch conus branch (cb), and left circumflex branch (cx). E) Left septal artery (sa). The right ventricular free color in E'). Peripheries of the left septal artery penetrated the ventricular free wall to distribute onto the

Figure 2

310 Cardiac veins. A) Middle cardiac vein (mcv). In this sample, the middle cardiac vein receives the left circumflex vein (cx) and small cardiac vein (scv). B) The great cardiac vein consists of the anterior 
313 the left atrium and great arteries to empty the right atrium behind the aortic bulb (open arrowhead). C)

314 Anterior cardiac vein (acv) drains from the anterior right ventricular wall and empties into the base of the

315 right auricle (open arrowhead). A, E7.25 (stage 32) heart; B, E8.0 (stage 35); C, E7.0 (stage 32); acv,

316 anterior cardiac vein; aivs, anterior interventricular segment of great cardiac vein; Ao, aorta; bs, basal

317 segment of great cardiac vein; cx, left circumflex vein; LV, left ventricle; Pt, pulmonary trunk; RV, right

318 ventricle; scv, small cardiac vein; Bar, $500 \mu \mathrm{m}$.

\section{Figure 3}

321 Schematic showing the coronary arteries and cardiac veins of the fetal quail heart. A) The major coronary arteries are the right and left septal arteries (rsa and lsa), which respectively originate from the right and left coronary stems. The right septal artery pierces the supraventricular crista and left septal artery infundibulum to enter the ventricular septum (gray color). Peripheries of the right and left septal arteries penetrate the ventricular free wall at the posterior and anterior interventricular sulci, respectively, and distribute over the ventricular surface. B) The cardiac veins consist of three major veins according to their openings into the right atrium, the middle cardiac vein (mcv), great cardiac vein, and anterior cardiac vein (acv). The most characteristic feature of the quail cardiac veins is the course of the great cardiac vein, which ascends along the anterior interventricular sulcus (anterior interventricular segment, aivs) and then courses between the left atrium and great arteries (basal segment, bs) to open into the right atrium. Note that panel A shows the ventricular septum seen form the right ventricle after removing the right 
332 ventricular free wall and panel B shows the cardiac base after removing the atria. acv, anterior cardiac

333 vein; aivs and bs, anterior interventricular segment and basal segment of the great cardiac vein; Ao, aorta;

334 lcx, left circumflex branch; lsa, left septal artery; mcv, middle cardiac vein; MV, mitral valve; Pt,

335 pulmonary trunk; rcx, right circumflex branch; rsa, right septal artery; TV, tricuspid valve.

336 
Table 1 Number of hearts examined

\begin{tabular}{ccccccccccc}
\hline E & 6 & 7 & 8 & 9 & 10 & 11 & 12 & 13 & 14 & Total \\
HH & $29-30$ & $32-33$ & 35 & $36-37$ & $38-39$ & 40 & 42 & 42 & 43 & 82 \\
\hline Artery & 4 & 4 & 2 & 11 & 11 & 29 & 5 & 6 & 10 & 82 \\
Vein & 4 & 3 & 3 & 2 & 3 & 5 & & & \\
\hline
\end{tabular}

E, embryonic day; HH, Hamburger and Hamilton stage adapted into quail. 
Right coronary branches
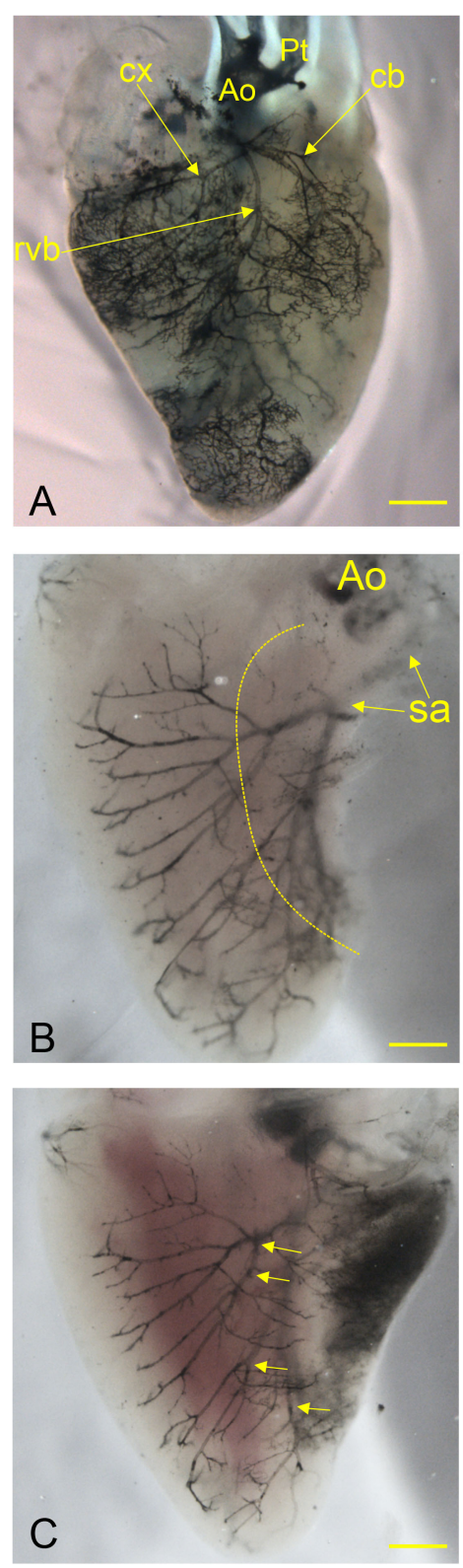

Anterior surface

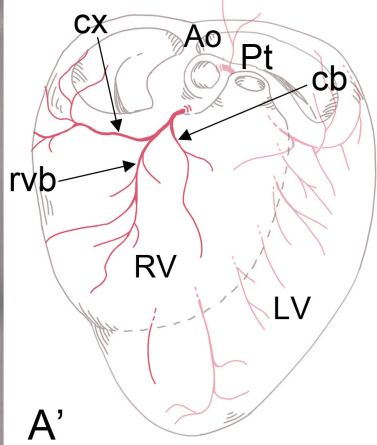

Ventricular septum

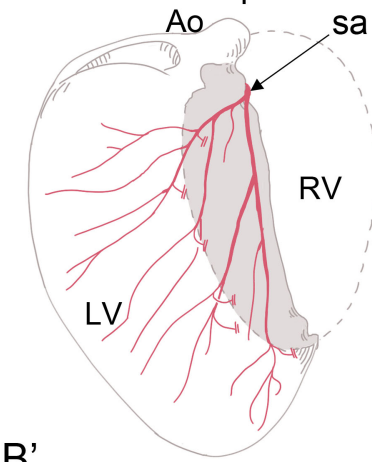

B'
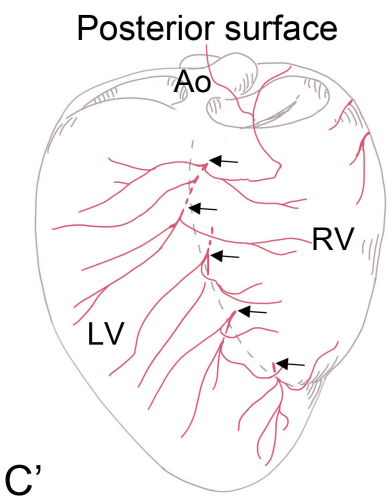

Left coronary branches

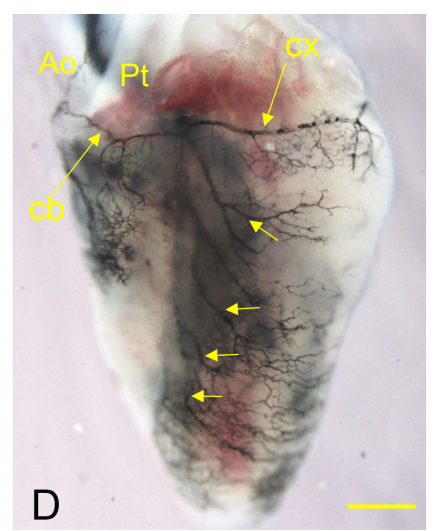

Anterior surface
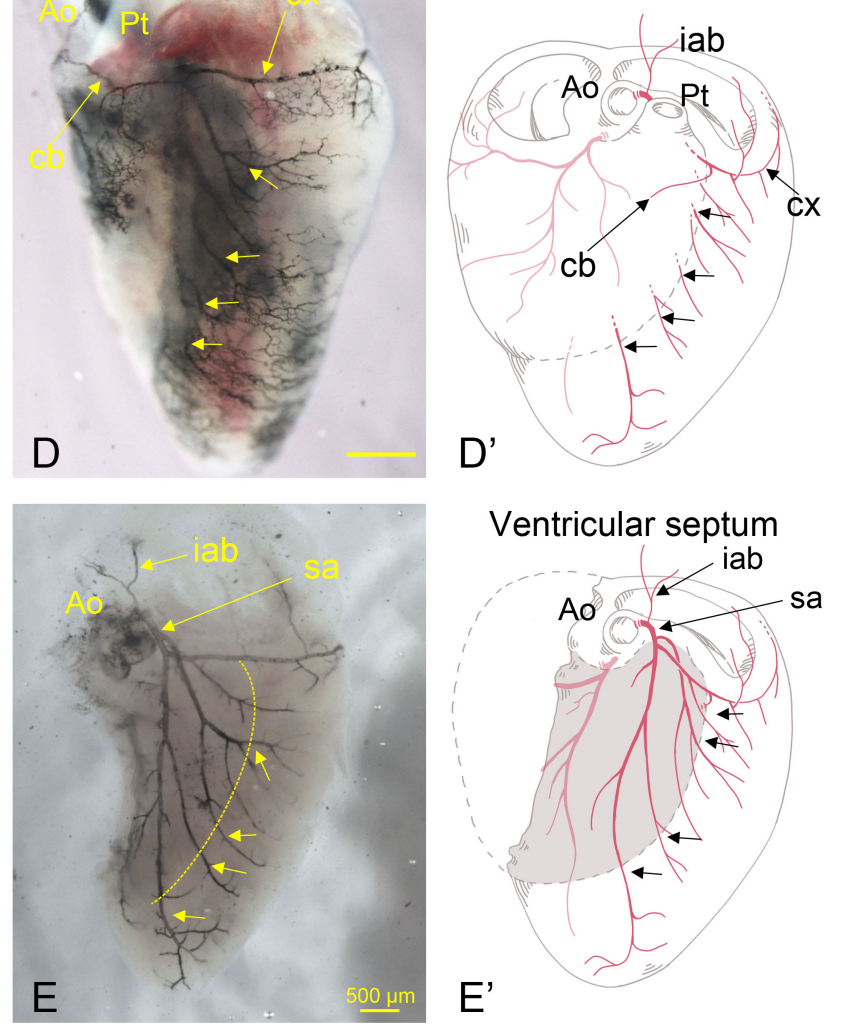

$E^{\prime}$

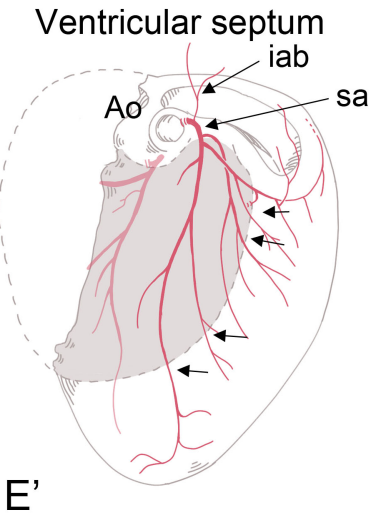



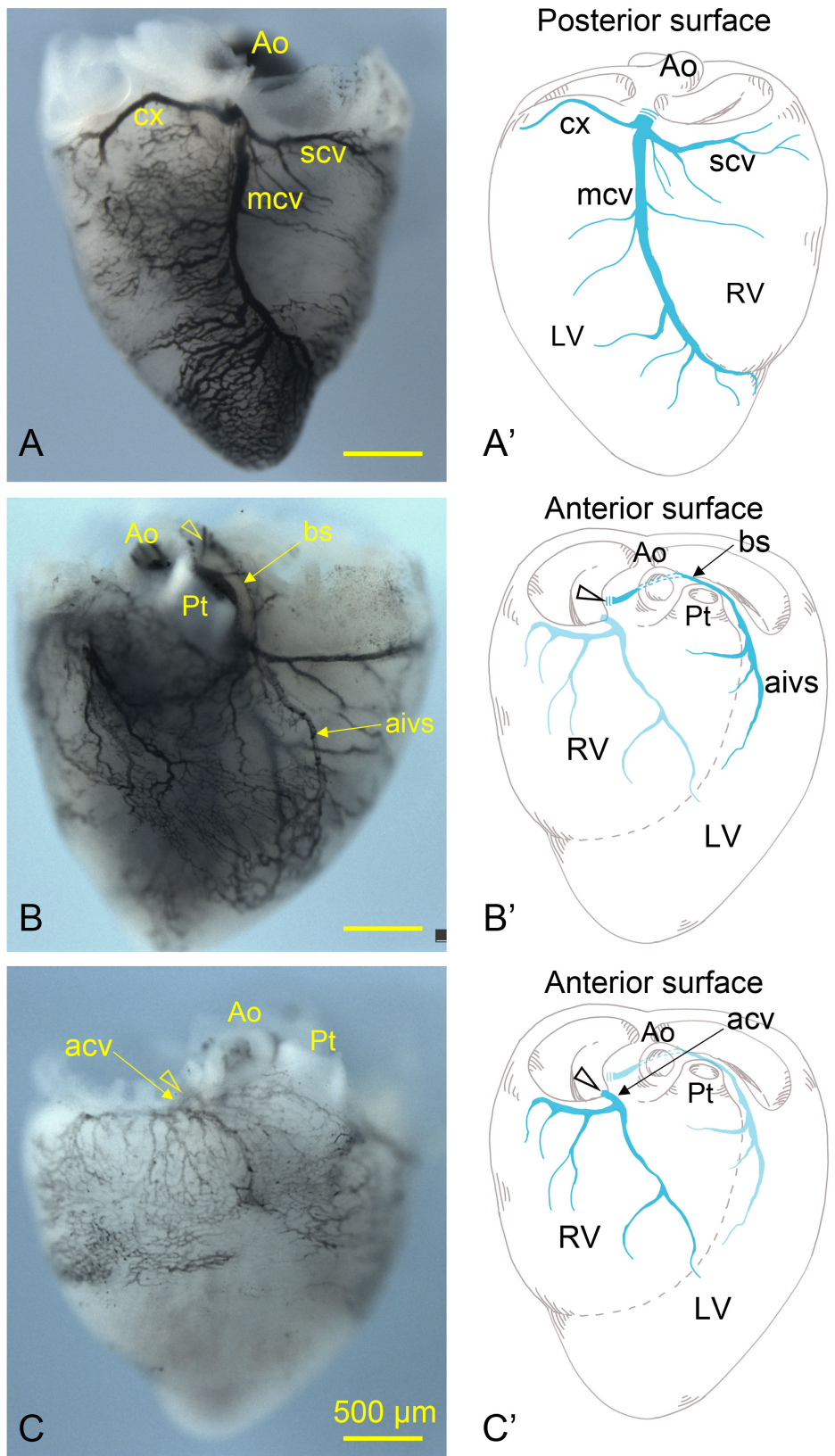

Anterior surface

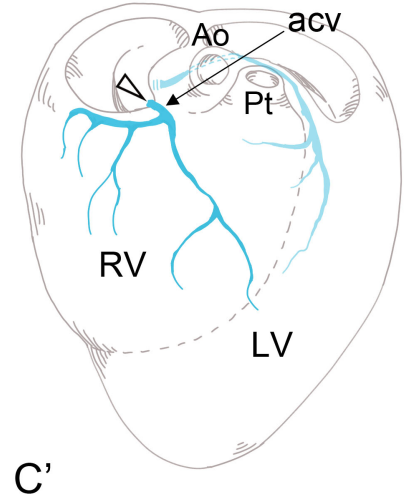


A

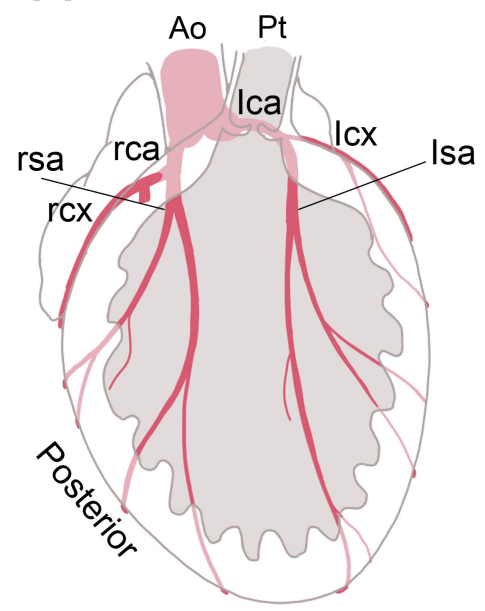

B

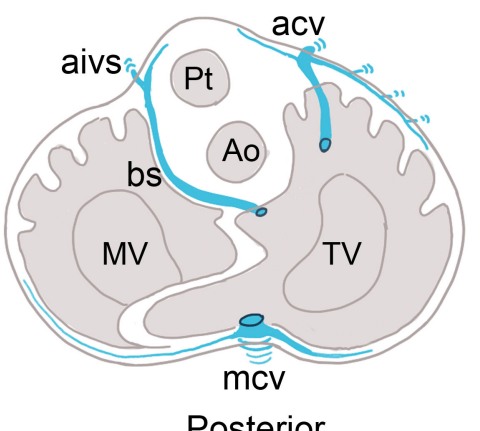

